\title{
A New Method for Skeleton Pruning
}

\author{
Laura Alejandra Pinilla-Buitrago, José Fco. Martínez-Trinidad, \\ and J.A. Carrasco-Ochoa \\ Instituto Nacional de Astrofísica, Óptica y Electrónica \\ Departamento de Ciencias Computacionales \\ Luis Enrique Erro \# 1, Puebla, México \\ \{laurapin, fmartine, ariel\}@inaoep.mx
}

\begin{abstract}
A Skeleton is a simplified and efficient descriptor for shapes, which is of great importance in computer graphics and vision. In this paper, we present a new method for computing skeletons from 2D binary shapes. The contour of each shape is represented by a set of dominant points, which are obtained by a nonparametric method. Then, a set of convex dominant points is used for building the skeleton. Finally, we iteratively remove some skeleton branches in order to get a clean skeleton representation. The proposed method is compared against other methods of the state of the art. The results show that the skeletons built by our method are more stable across a wider range of shapes than the skeletons obtained by other methods; and the shapes reconstructed from our skeletons are closer to the original shapes.
\end{abstract}

Keywords: skeleton, pruning, distance transform, binary shapes.

\section{Introduction}

Skeleton based representation has several advantages at reducing dimensionality and capturing geometrical and topological properties of shapes [1].

Many definitions of skeleton have been reported in the literature. One of the most accepted is the Medial Axis Transform (MAT) proposed by H. Blum. In MAT, a skeleton is a set of points, where each point is the center of a maximal disk inside object boundaries. The algorithms developed for skeleton extraction can be classified into five categories: direct simulation of grassfire model, analytical computation, iterative thinning with topology preserving, Voronoi diagram based, and distance transform based [2-3].

The representation of shapes by skeletons has shown being useful in tasks of object recognition and classification. However, in those cases where there are large deformations and intra-class variations of objects, boundary noise or small perturbations in the shapes, the skeleton obtained using conventional methods is usually accompanied with too many superfluous branches, and it affects the performance of object recognizers [3]. For this reason, some recently proposed algorithms for representation of shapes by skeletons include prunes of spurious branches. 
In this work, we develop a new method based on the works proposed by Montero [4], Hesselink [5] and Prassad [6], in order to obtain a stable skeleton without unwanted branches.

This paper is organized as follows: In Section 2, we review the related work. In Section 3, we describe the proposed method. Section 4 shows experimental results of the proposed method compared with other state of the art methods. Finally, conclusions and future work are shown in Section 5.

\section{Related Work}

In the literature, there are many approaches for computing and pruning skeletons from binary shapes [2-4, 7-11]. During the process of skeletonization usually appear unwanted branches because of digital noise and deformation in the shapes; therefore pruning is an essential stage in skeletonization algorithms.

Several pruning methods into skeletonization algorithms have been proposed, which can be categorized in three main categories: assigning significance values to skeleton points and removing insignificant points according to a given threshold; preprocessing the shape's boundary (e.g., boundary smoothing, find knots or dominant points); and removing unwanted branches [7]. The methods of the first category could produce disconnected skeletons, breaking the skeleton into pieces or they cannot completely remove insignificant branches. The second kind of methods aims to remove unwanted boundary noise, but it could change the original shape and consequently shifting the skeleton and its topological properties. The methods in the last category prune the skeleton branch-by-branch taking into account local or global information, using significance measures of the skeleton branches [3-4, 7-10].

Until now, it does not already exist a non-parametric method or a method with parameters invariant for any shape that allows generating skeletons identical to handlabeled shapes. However, in the literature, we can find some methods requiring less input parameters than others and sometimes the input values are independent for a wide range of shapes.

\section{Proposed Method}

The proposed method combines the non-parametric method for dominant point detection introduced in [6], with the skeleton construction and a modification of the pruning process presented in [4-5]. The proposed method is divided into three stages: piecewise curve segmentation, skeleton computation and skeleton pruning (Fig. 1).

In the first stage, we find the dominant points located in the shape with the method proposed in [6] (section 3.1). This is a non-parametric method which has as main advantage for our method that it does not need thresholds or input parameters. According to the literature, this method is considered as the only one non-parametric method for finding dominant points (see (a1) in Fig. 1). After, from the set of 
dominant points we obtain the convex points (see (a2) in Fig. 1), and unlike Montero and Lang in [4], we only use the set of convex dominant points to split piecewise curve split (see (a3) in Fig. 1).

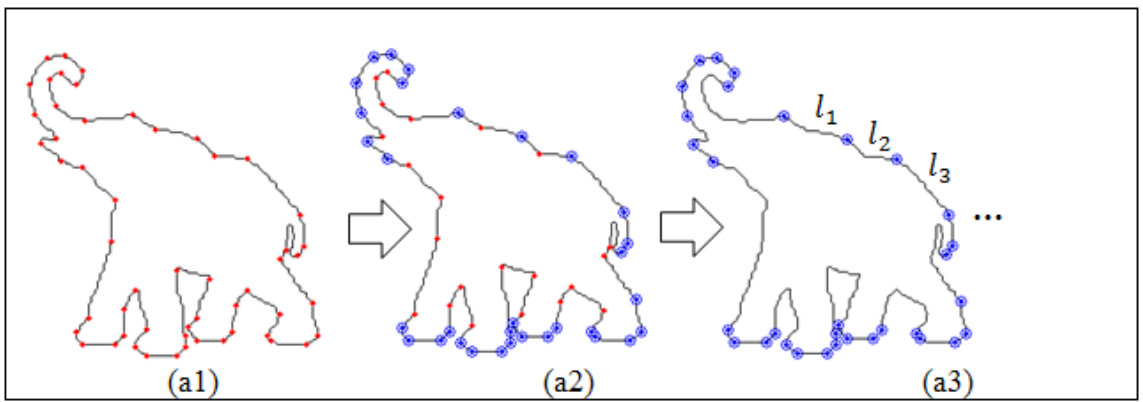

(a)

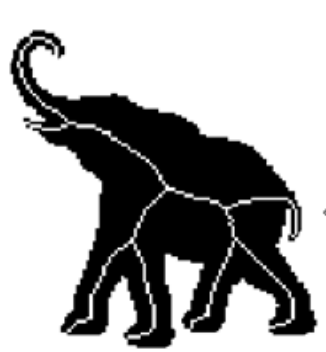

(c)

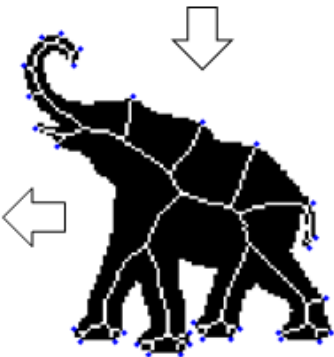

(b)

Fig. 1. (a) Curve segmentation by piecewise: (a1) Dominant point detection with a nonparametric method. (a2) Set of convex dominant points. (a3) Splitting of piecewise curve (b) Skeleton computation. (c) Skeleton pruning.

The piecewise curve is used to compute the skeleton by means of the integer medial axis (IMA) [4-5]. Our method produces a first approximation to the pruned skeleton (see (b) in Fig. 1) by selecting pixels as skeleton points. Skeleton construction by means of IMA considers a distance transform. The distance transform $D(x, y)$ is a function, which computes the distance from any point inside the shape to the closest point on the shape boundary.

In the last stage (see (c) in Fig. 1), we use a pruning criterion (Section 2.2) similar to the one proposed in [4] but we add additional conditions to ensure a more stable skeleton for a wide range of shapes using a single threshold. Moreover, in our experiments, several shapes belonging to different classes showed good results using the same threshold for the entire set of shapes, compared with the results obtained by other methods, which mostly need to specify parameter values for each shape.

\subsection{Non-parametric Method for Dominant Point Detection}

Since polygonal approximation allows representing a digital curve using less of points, some methods for building skeletons approximate the shape by polygons, 
either for removing noise as a previous step to the construction of the skeleton or for building the skeleton using the vertices of the polygons $[4,10]$.

Most of the methods for approximating shapes by obtaining dominant points require some of the following conditions: a) the number of vertices for the polygonal approximation, such that the error distance between shape's boundary and the polygon is minimal; b) a threshold defining the maximum distance error allowed between the contour and the polygonal approximation, such that a minimum number of points would be obtained for representing the boundary.

Ramer, Douglas and Peucker (RDP) [12, 13] propose a recursive method for reducing the number of points in a curve, which are called dominant points. Consider a set of edge points defining a curve $e=\left\{P_{1}, P_{2}, \ldots, P_{N}\right\}$, where $P_{i}$ is the $i$ th edge point in the digital curve $e$. The line passing through a pair of points $P_{a}\left(x_{a}, y_{a}\right)$ and $P_{b}\left(x_{b}, y_{b}\right)$ is given by:

$$
x\left(y_{a}-y_{b}\right)+y\left(x_{b}-x_{a}\right)+y_{b} x_{a}-y_{a} x_{b}=0
$$

Then the deviation $d_{i}$ of a pixel $P_{i}\left(x_{i}, y_{i}\right) \in e$ from the line passing through the pair $\left\{P_{1}, P_{N}\right\}$ is given by:

$$
d_{i}=\frac{\left|x_{i}\left(y_{1}-y_{N}\right)+y_{i}\left(x_{N}-x_{1}\right)+y_{N} x_{1}-y_{1} x_{N}\right|}{\sqrt{\left(x_{N}-x_{1}\right)^{2}+\left(y_{1}-y_{N}\right)^{2}}}
$$

Thus, the point with the maximal deviation is found and denoted as $P_{\text {max }}$; it allows to obtain new segments of the curve. This process is repeated until:

$$
\max \left(d_{i}\right)<d_{t o l}
$$

As we can see, this method requires a threshold $d_{t o l}$. For making this method a non-parametric method, Prasad et al., in [6] consider the maximum deviation of the digital curve as an error function related to the quality of fitting, allowing the threshold to be automatically and adaptively obtained (see Fig. 2).

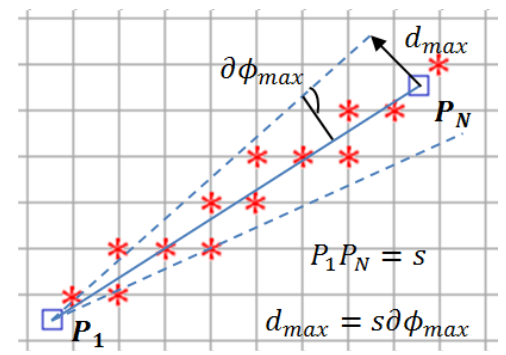

Fig. 2. The maxim deviation $d_{\max }$ of a line segment [6]

If a line segment in the continuous 2-dimensional space is digitized, the maximum difference between the angles made by the digital line segment and the continuous line segment with respect to the $x$-axis is given by: 


$$
\begin{gathered}
\delta \emptyset_{\text {max }} \approx \max \left(\tan ^{-1}\left\{\frac{1}{s}(|\sin \emptyset \pm \cos \emptyset|)\left(1-t_{\text {max }}+t_{\text {max }}^{2}\right)\right\}\right) \\
\varnothing=\tan ^{-1}(m) \\
t_{\text {max }}=\left(\frac{1}{s}\right)(|\cos \emptyset|+|\sin \emptyset|) \\
d_{t o l}=s \partial \emptyset_{\text {max }} \approx \max \left(s \tan ^{-1}\left\{\frac{1}{s}(|\sin \emptyset \pm \cos \emptyset|)\left(1-t_{\max }+t_{\text {max }}^{2}\right)\right\}\right)
\end{gathered}
$$

Where $\emptyset$ is the slope $m$ of the continuous line segment $P_{1} P_{N}$ and $s$ is the $P_{1} P_{N}$ distance.

\subsection{Final Pruning}

In our method, the two first stages allow us to obtain a skeleton with less unwanted branches in comparison to the skeleton obtained by conventional methods without pruning. Nevertheless, in order to obtain the desired final skeleton, some end branches need to be pruned.

Consider an end branch $\left\{b_{p}, \ldots, e_{p}\right\}$ with branch point $b_{p}$ and an end point $e_{p}$, such that there are not any other branch point or end point except $b_{p}$ and $e_{p}$, and let $f$ be the distance transform computed for each point in order to obtain the IMA skeleton.

We propose to prune a branch $\left\{b_{p}, \ldots, e_{p}\right\}$ if the Euclidean distance between the distance transform and the branch point $\left(\left|f-b_{p}\right|_{2}\right)$ with a scale factor $s$ is greater than the sum of the Euclidean distances between each pair of consecutive points conforming the branch $\left\{b_{p}, \ldots, e_{p}\right\}$, i.e.:

$$
\begin{gathered}
\sum_{i=1}^{n-1}\left|p_{i}-p_{i+1}\right|_{2} \leq s *\left|f-b_{p}\right|_{2} \\
\left\{b_{p}, \ldots, e_{p}\right\}=\left\{p_{1}, p_{2}, \ldots, p_{n}\right\} \text { Where } p_{1}=b_{p} \text { and } p_{n}=e_{p}
\end{gathered}
$$

This condition avoids pruning branches where the Euclidean distance $\left|e_{p}-b_{p}\right|_{2}$ is very close to the Euclidean distance between $\left|f-b_{p}\right|_{2}$, but the sum of Euclidean distances between every pair of consecutive points is much larger than $\left|e_{p}-b_{p}\right|_{2}$.

Additionally, when two or more end branches share the same junction point, we propose to delete just one end branch by iteration, eliminating only the shorter length branch. This condition, unlike the pruning proposed in [4], avoids losing structural information, as we will show in our experiments. 


\section{Experiments}

For comparing the proposed method, we used the skeletonization algorithms proposed by Zhang and Suen [11], Bai et al. [10], and Montero and Lang [4] using Bezier Curve approximation.

In a first experiment, we show the stability of the proposed method using the same parameter value in comparison to the other methods, displaying the skeletons obtained for several shapes (Fig. 3).

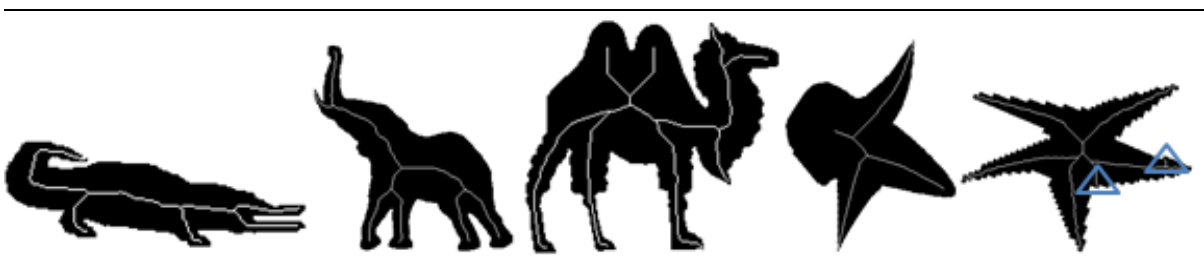

(a)

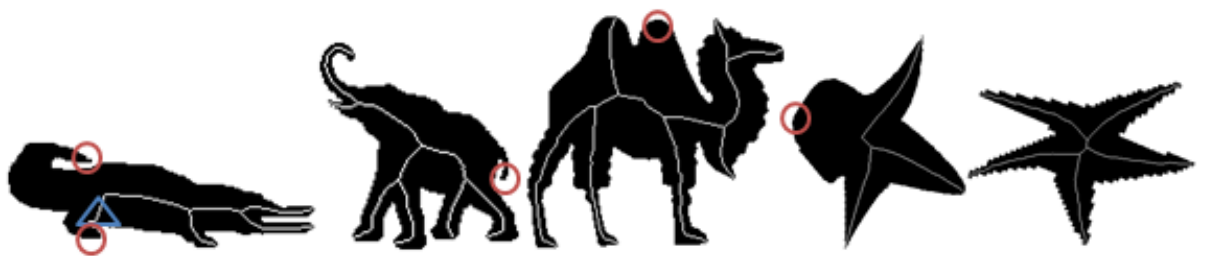

(b)
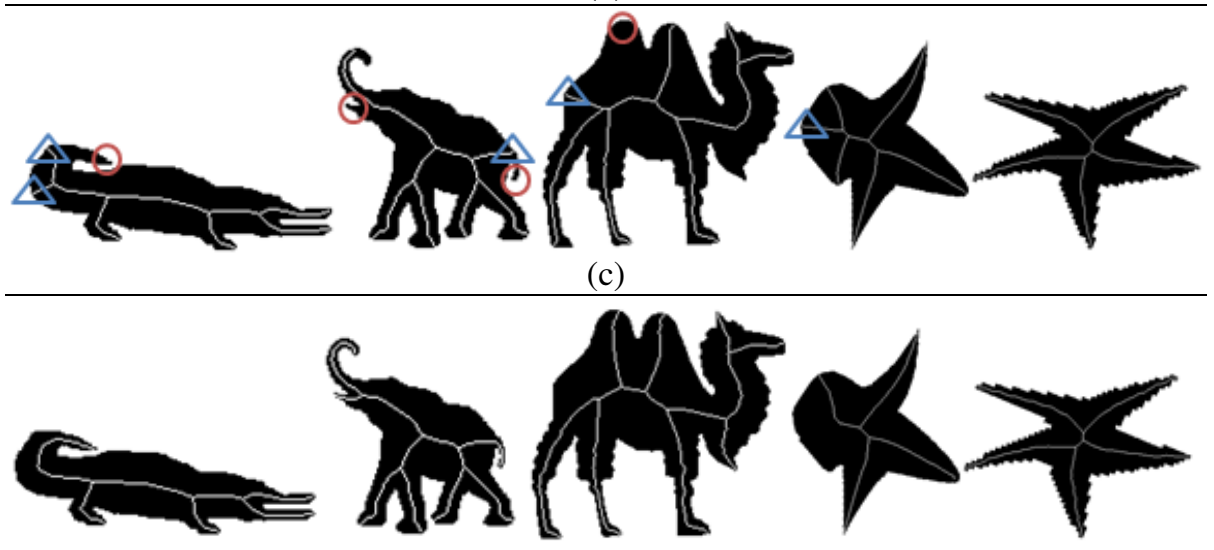

(d)

Additional branches

Missing branches

Fig. 3. Skeletons obtained with different methods: (a) Zhang and Suen [11]. (b) Bai et al. [10] for $N=10$. (c) Montero and Lang [4] approximation to boundary by cubic Bezier curves with $d=15$ and $s=1.25$ ( $s$ is a minimum distance between the boundary and fitting curve). (d) Proposed method with $s=1.27$. 
In the experiments of the Fig. 3, we use the same input parameter value for all the shapes in each tested method. The threshold used for each method was obtained experimentally; we use the trial-and-error method for choosing the parameter value, which obtains the best visual results for whole data set. From our first experiment, we can see that the proposed method produces more consistent and stable skeletons, unlike the other two methods, where some skeletons have unwanted or missing branches.

In Fig. 4, we can see that our method generates good representations for several shapes using the same threshold value. Unlike the Bai et al. [10] where the parameter values are dependent on the specific shape. Furthermore, the Montero and Lang method, although produces lower number of errors respect to the results obtained with [10], requires two parameter values unlike our proposed method that needs just only one. Although the Zhang and Suen method [11] produces few unwanted branches, the thinning method does not ensure that the points of the skeleton are centers of maximal disk, which affects the reconstruction of the shape.

In a second experiment, we compare the speed of the methods using the same shape. Furthermore, we compute the quality of the skeleton obtained by a quantitative evaluation using the Baseski and Tari dataset (1000 shapes).

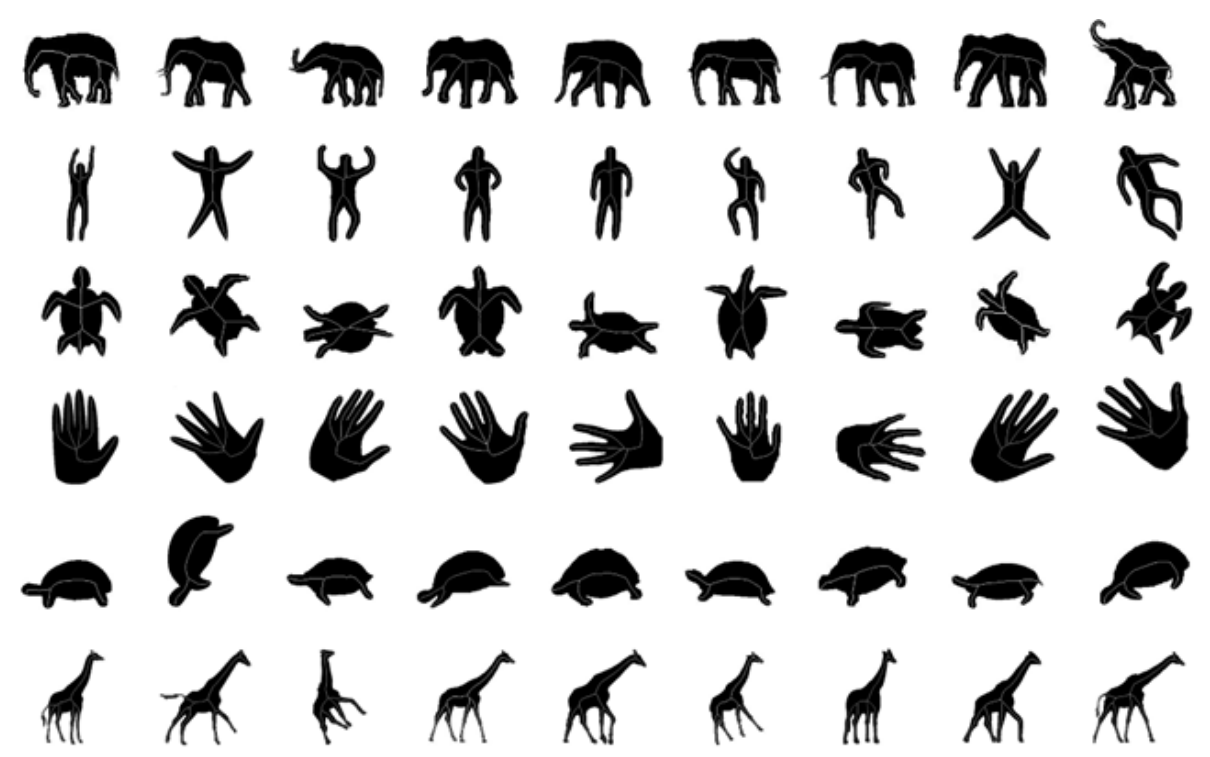

Fig. 4. Subset of shapes (Baseski and Tari dataset ) and their respective skeletons with the proposed method with $s=1.27$

One of the most common techniques for evaluating the quality of the skeletons obtained by algorithms for skeleton extraction is the reconstruction error ratio $(R E R)$. This measure evaluates the difference between the shape reconstructed through the skeleton and the original shape. The shape is reconstructed from the distance transform associated to each point of the skeleton. The $R E R$ is defined as: 


$$
R E R(S, A)=\frac{|\operatorname{Area}(\mathrm{A})-\operatorname{Area}(\mathrm{R}(\mathrm{S}))|}{\operatorname{Area}(\mathrm{A})}
$$

Where $S$ is a skeleton of the shape $A$ and $R(S)$ is the shape reconstructed from $S$, Area(.) denotes the area of a shape measured in pixels.

For comparing the quality of the obtained skeletons, in the table 1 we show the execution time and skeleton quality for an image chosen from the Baseski and Tari dataset. Where we can see that our method obtains better $R E R$ result and visually generates the best reconstructed shape, preserving the topology from the original shape.

Table 1. Comparison of the proposed method against the skeletons obtained by Zhang and Suen [11], Bai et al. [10] and Montero [4] methods in terms of the runtime and quality of the skeletons. The reconstructed shapes are red and the reconstruction errors are marked with black.

\begin{tabular}{|c|c|c|c|c|}
\hline \multicolumn{2}{|c|}{ [324×343] } & Runtime & $\begin{array}{c}\text { Skeleton } \\
\text { quality }\end{array}$ & Reconstructed \\
\hline $\begin{array}{c}\text { Zhang and Suen } \\
{[11]}\end{array}$ & -- & 2.6399 & 0.0560 & \\
\hline Bai et al. [10] & $\mathrm{N}=10$ & 2.2068 & 0.0663 & \\
\hline $\begin{array}{c}\text { Montero and Lang } \\
\text { [4] }\end{array}$ & $\begin{array}{c}d=15 \\
s=1.25\end{array}$ & 1. 2534 & 0.0709 & \\
\hline Proposed method & $s=1.27$ & 1.6842 & 0.0281 & \\
\hline
\end{tabular}


In the table 2, we show the average for reconstruction error ratio and time execution for a whole dataset containing 1000 shapes (Baseski and Tari dataset), where we can see that our method obtains in average the best RER result.

The tests were carried out in a computer with the following characteristics: processor Intel core i5 at $2.30 \mathrm{GHz}, 4 \mathrm{~Gb}$ of RAM, 64-bit Windows operating system. As it can be seen from tables 1 and 2, our method did not get the best runtime in comparison with the other methods, but it is very close to the best runtime obtained by Montero's method (the fastest).

Table 2. Average runtime and reconstruction error ratio of skeletons for the Baseski and Tari dataset over 1000 shapes

\begin{tabular}{|c|c|c|c|}
\cline { 3 - 4 } \multicolumn{2}{c|}{} & $\begin{array}{c}\text { Average execution } \\
\text { time (Seconds) }\end{array}$ & $\begin{array}{c}\text { Average skeletons } \\
\text { quality (RER) }\end{array}$ \\
\hline Zhang and Suen [11] & -- & 2.6488 & 0.0817 \\
\hline Bai et al. [10] & $N=10$ & 2.5092 & 0.0392 \\
\hline $\begin{array}{c}\text { Montero and Lang } \\
{[4]}\end{array}$ & $\begin{array}{c}s=1.25 \\
d=15\end{array}$ & $\mathbf{0 . 8 2 5 9}$ & 0.0504 \\
\hline Proposed method & $s=1.27$ & 1.1794 & $\mathbf{0 . 0 2 6 4}$ \\
\hline
\end{tabular}

\section{Conclusions}

In this work, we present a new method for computing skeletons from 2D binary shapes. The contour of each shape is represented by a set of dominant points, which are obtained by a nonparametric method. Then, a set of convex dominant points is used for building the skeleton. Finally, we introduce some new conditions in the pruning stage that allow iteratively removing some skeleton branches in order to get a clean skeleton representation.

From our experiments, we can see that in the proposed method the same threshold value works fine for several types of shapes; it is reflected in the fact that the skeleton's quality is clearly better in comparison to other methods widely used in the literature. Our experiments also show that our method allows representing shapes by simple and stable skeletons for a wider range of shapes than other methods.

As future work, we propose study the inclusion of the whole contour information for obtaining more consistent skeletons.

Acknowledgment. This work was partly supported by the National Council of Science and Technology of Mexico (CONACyT) through the project grants CB2008-106443 and CB2008-106366; and the scholarship grant 283120.

\section{References}

1. Bai, X., Latecki, L.J.: Path Similarity Skeleton Graph Matching. IEEE Transactions on Pattern Analysis and Machine Intelligence 30(7) (2008) 
2. Duan, H., Wang, J., Liu, X., Liu, H.: A Skeleton Pruning Approach Using Contour Length as the Significance Measure. In: Third International Conference on Pervasive Computing and Applications, ICPCA, vol. 1, pp. 360-364, 6-8 (2008)

3. Shen, W., Bai, X., Hu, R., Wang, H., Latecki, J.L.: Skeleton growing and pruning with bending potential ratio. Pattern Recognition 44, 196-209 (2011)

4. Montero, A.S., Lang, J.: Skeleton pruning by contour approximation and the integer medial axis transform. Computers and Graphics 36, 477-487 (2012)

5. Hesselink, W.H., Roerdink, B.T.M.: Euclidean Skeleton of Digital Image and Volume Data in Linear Time by the Integer Medial Axis Transform. IEEE Transactions on Pattern Analysis and Machine Intelligence 30(12) (2008)

6. Prasad, D.K., Quek, C., Leung, M.K.H., Cho, S.: A novel framework for making dominant point detection methods non-parametric. Image and Vision Computing (Elsevier) 30(11), $843-859$ (2012)

7. Liu, H., Wu, Z., Zhang, X., Hsu, D.F.: A skeleton pruning algorithm based on information fusion. Pattern Recognition Letters 34, 1138-1145 (2013)

8. Liu, H., Wu, Z., Hsu, S.F., Peterson, B.S., Xu, D.: On the generation and pruning of skeletons using generalized Voronoi diagrams. Pattern Recognition Letters 33, 2113-2119 (2012)

9. Duan, H., Wang, J., Liu, X., Liu, H.: A Skeleton Pruning Approach Using Contour Length as the Significance Measure. In: Third International Conference on Pervasive Computing and Applications, ICPCA 2008, vol. 1, pp. 360-364 (2008)

10. Bai, X., Latecki, J.L., Liu, W.: Skeleton pruning by contour partitioning with Discrete Curve Evolution. IEEE Transactions on Pattern Analysis and Machine Intelligence 29(3), 449-462 (2007)

11. Zhang, T.Y., Suen, C.Y.: A fast parallel algotithm for thinning digital patterns. ACM Commun. 27, 236-239 (1984)

12. Douglas, D.H., Peucker, T.K.: Algorithms for the Reduction of the Number of Points Required to Represent a Digitized Line or its Caricature. Cartographica: The International Journal for Geographic Information and Geovisualization, 112-122 (1973)

13. Ramer, U.: An iterative procedure for the polygonal approximation of plane curves. Computational Graphics Image Process, 244-256 (1972) 